Thomas Wex

Der Nonprofit-Sektor der Organisationsgesellschaft 


\section{GABLER EDITION WISSENSCHAFT}

NPO-Management

Herausgegeben von

Professor Dr. Dieter Witt

Technische Universität München

Dienstleistungsökonomik

mit Seminar für Vereins- und

Verbandsforschung (SVV)

Das Management von Non-Profit-Organisationen (NPO), insbesondere des Dritten Sektors - neben Staat und Privatwirtschaft -, wird zunehmend von der betriebswirtschaftlichen Forschung untersucht. In dieser Schriftenreihe werden wichtige Forschungs- und Diskussionsbeiträge zu diesen gemein- oder bedarfswirtschaftlichen Betrieben präsentiert, die von Verbänden, Vereinen, Stiftungen, öffentlichen Betrieben bis zu Großhaushalten reichen. Die Veröffentlichungen wenden sich gleichermaßen an Theoretiker und Praktiker. 


\author{
Thomas Wex
}

\title{
Der Nonprofit-Sektor der Organisationsgesellschaft
}

Mit einem Geleitwort von Prof. Dr. G. Günter Voß

Deutscher Universitäts-Verlag 
Bibliografische Information Der Deutschen Bibliothek

Die Deutsche Bibliothek verzeichnet diese Publikation in der Deutschen

Nationalbibliografie; detaillierte bibliografische Daten sind im Internet über

$<$ http://dnb.ddb.de> abrufbar.

Dissertation Technische Universität Chemnitz, 2002

\section{Auflage März 2004}

Alle Rechte vorbehalten

(C) Deutscher Universitäts-Verlag/GWV Fachverlage GmbH, Wiesbaden 2004

Lektorat: Brigitte Siegel / Nicole Schweitzer

Der Deutsche Universitäts-Verlag ist ein Unternehmen von

Springer Science+Business Media.

www.duv.de

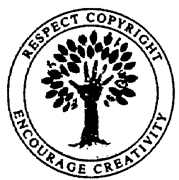

Das Werk einschließlich aller seiner Teile ist urheberrechtlich geschützt. Jede Verwertung außerhalb der engen Grenzen des Urheberrechtsgesetzes ist ohne Zustimmung des Verlags unzulässig und strafbar. Das gilt insbesondere für Vervielfältigungen, Übersetzungen, Mikroverfilmungen und die Einspeicherung und Verarbeitung in elektronischen Systemen.

Die Wiedergabe von Gebrauchsnamen, Handelsnamen, Warenbezeichnungen usw. in diesem Werk berechtigt auch ohne besondere Kennzeichnung nicht zu der Annahme, dass solche Namen im Sinne der Warenzeichen- und Markenschutz-Gesetzgebung als frei zu betrachten wären und daher von jedermann benutzt werden dürften.

Umschlaggestaltung: Regine Zimmer, Dipl.-Designerin, Frankfurt/Main

Gedruckt auf säurefreiem und chlorfrei gebleichtem Papier

ISBN-13:978-3-8244-7940-5

e-ISBN-13:978-3-322-81608-5

DOI: $10.1007 / 978-3-322-81608-5$ 


\section{Geleitwort}

„Ehrenamt“, „Nonprofit Organisationen“, „Dritter Sektor“, „,bürgerschaftliches Engagement“seit einiger Zeit Vokabeln mit Konjunktur. Lange war eine Tätigkeit außerhalb der formellen Erwerbstätigkeit bzw. in nicht unmittelbar privatwirtschaftlichen oder staatlichen Bereichen der Gesellschaft ein vernachlässigtes Feld der wissenschaftlichen und öffentlichen Diskussion. Nur einige versprengte Spezialisten aus der Sozialpädagogik, der politologischen und ökonomischen Vereins- und Verbändeforschung, der Frauenforschung oder aus betroffenen Organisationen und Initiativen bemühten sich um dieses Thema.

Aber jetzt sind Aktivitäten in der Gesellschaft, die weder privatwirtschaftliche noch staatliche, aber auch nicht im engeren Sinne ,private“ (Haus- und Familien-) Tätigkeiten sind, in aller Munde. Sogar der Bundesregierung ist dieses Thema eine breit angelegte Initiative wert. Und man fragt sich, was dahinter steckt. Zwei ineinander greifende Entwicklungen spielen dabei offensichtlich eine wichtige Rolle:

Zum einen gibt es immer mehr Menschen, die eine persönlich und gesellschaftlich sinnvolle Betätigung jenseits von Erwerbsarbeit in der profitorientierten Ökonomie oder in staatlichen Organisationen suchen und sich dabei nicht mit rein privaten Aktivitäten (etwa in der Familie) zufrieden geben. Sie wollen eine befriedigende und nützliche Aufgabe in der Öffentlichkeit wahrnehmen, die nicht direkt ökonomischen oder staatlichen Vorgaben und Zwängen unterliegt.

Zum anderen haben die Öffentlichkeit und insbesondere die Politik zunehmend entdeckt, dass es in diesem Sektor ein großes Potential von aktiven Menschen gibt, die bereit sind, auch ohne umfangreiche Entlohnung gesellschaftlich nützliche Funktionen zu übernehmen. Ein Potential, das dazu genutzt werden kann, gesellschaftliche Aufgaben flexibler und problemnäher, vor allem aber auch kostengünstiger zu bearbeiten. Eine willkommene Perspektive in Zeiten knapper öffentlicher Kassen.

Beide Entwicklung führten dazu, dass das bisher eher vernachlässigte beachtete Feld wachsende Aufmerksamkeit erhielt. Man registrierte vor allem auch in der Wissenschaft, dass die Kenntnisse über diesen durchaus gewichtigen Bereich der Gesellschaft reichlich dürftig waren. Man erkannte, wie wenig man über die Größenordnungen, die Erscheinungsformen, die gesellschaftlichen Funktionen und Beziehungen usw. des „Nonprofit-Sektors“ und über die dort ausgeübten vermeintlich ,ehrenamtlichen“ oder „freiwilligen“ Tätigkeiten wusste. Und man merkte auch, dass schon die üblichen Begriffe („Non-Profit", „Ehre“ usw.), wenn man sie etwas aufmerksamer betrachtete, darauf hinwiesen, wie ungenau das Verständnis von dem damit bezeichneten Feld war. Zu wissen, dass ein Feld von Tätigkeiten etwas „nicht“ hat, was 
ein anderes ausmacht, nämlich profitorientiert zu sein, reichte nicht mehr - auch nicht, dass ein großer Bereich der Gesellschaft ein ,dritter“ neben zwei anderen ist, mit denen man sich seit langem intensiv beschäftigt. Kurz: der empirische wie theoretische Forschungsbedarf wurde überdeutlich.

Der Autor dieses Bandes gehört seit Jahren zu den wenigen Wissenschaftlern, die sich nicht nur selbst unmittelbar im Nonprofit-Bereich engagieren, sondern auch dafür einsetzen, diesen (fast) vergessenen Bereich systematisch einer wissenschaftlichen Bearbeitung zuzuführen.

Mit seiner nun vorliegenden Studie legt Thomas Wex nicht nur ein umfassendes Kompendium der einschlägigen Forschung mit besonderer Berücksichtigung der bisherigen theoretischen Bemühungen um den Gegenstand vor, was sowohl der Praxis wie der einschlägigen Wissenschaft von großem Nutzen sein wird.

Er präsentiert darüber hinaus einen überzeugenden Vorschlag, wie die innere „Logik“ von „,nonprofit-orientierten“, „ehrenamtlichen“, freiwilligen“ Tätigkeitsfeldern in der Gesellschaft sozialwissenschaftlich gefasst werden kann: Geht es in der Wirtschaft um ökonomischen Zugewinn und ist der Staat durch die Anwendung von (rechtlich kodifizierter) Macht gekennzeichnet, so wird der „Dritte“ oder „Nonprofit- Sektor“ dadurch gekennzeichnet, dass sich hier Menschen unmittelbar zur Bearbeitung gesellschaftlicher Probleme zusammenfinden - die spezifische Qualität dieses Bereichs besteht darin, dass sich die Beteiligten aktiv, selbstbestimmt und direkt aufgabenbezogen ,, assoziieren“. Ich bin mir sicher, dass dieses konzeptionelle Angebot eine befruchtende und klärende Wirkung auf die (Weiter-) Entwicklung der Theoriearbeit zum Thema „Dritter-Sektor“ haben wird.

Die Arbeit von Thomas Wex wurde mit dem Universitätspreis 2003 der Technischen Universität Chemnitz ausgezeichnet. Ich wünsche dem spannenden Buch, das als ein bedeutender Beitrag zur Nonprofit-Forschung angesehen werden kann, viel Erfolg und vor allem viele interessierte Leser.

G. Günter Voß 


\section{Vorwort}

„Gibt es einen Dritten Sektor?“, war die nun schon mehr als ein Jahrzehnt zurückliegende viel zitierte Frage Wolfgang Seibels zu Beginn der deutschen Beschäftigung mit dem NonprofitSektor als Forschungsobjekt. ${ }^{1}$ Obwohl seitdem eine Vielzahl von Arbeiten erschienen, die eine Bejahung dieser Frage erlauben, steht die Nonprofit-Forschung dennoch erst am Anfang. Der Kenntnisstand über die beiden anderen Grundtypen von Organisationen: staatliche Behörden/ Verwaltungen und Wirtschaftsunternehmen, und ihre jeweiligen Organisationssektoren: Staat und Erwerbswirtschaft, ist noch ungleich größer als der zu Nonprofit-Organisationen und dem Nonprofit-Sektor.

Die vorliegende Arbeit möchte dazu beitragen diese Lücke zu schließen. Sie verfolgt das Ziel, das Projekt voranzutreiben, den Nonprofit-Sektor als einen distinkten dritten Organisationsbereich der Gesellschaft zu begründen. Der Sektor wird im folgenden Text als ein gesellschaftliches Teilsystem von Organisationen konzeptualisiert, dessen »Logik« im Gegensatz zu Erwerbswirtschaft und Staat in seinem assoziativen, kooperativen Charakter liegt.

Die Arbeit beinhaltet hierzu drei große Teile, die bislang in der Nonprofit-Forschung in dieser Form nicht behandelt wurden: Eine historische Beschreibung der Entstehung und Entwicklung der Organisationsformen, insbesondere der Nonprofit-Organisationen. Ein empirisches und theoretisches Modell der Organisationsgesellschaft mit seinen Organisationstypen und drei Organisationssektoren, worin die Nonprofit-Organisationen verortet werden, sowie die Rekonstruktion einer »Organisationslogik« der Nonprofit-Organisation oder hier so bezeichneten "Systemrationalität« des gesellschaftlichen Teilsystems Nonprofit-Sektor. Die Arbeit leistet somit einen Beitrag zur Diskussion der drei zentralen Fragestellungen der Nonprofit-Forschung ohne diese abschließend beantworten zu wollen: Warum gibt es Nonprofit-Organisationen? Inwieweit unterscheiden sich Nonprofit-Organisationen von anderen Organisationstypen? Welche Position nimmt der Nonprofit-Sektor in der Gesamtstruktur der Gesellschaft ein? ${ }^{2}$

Das Interesse an Sphären oder Aktivitäten jenseits von Erwerbswirtschaft (»Markt«) und Staat hat in den letzten Jahren stark zugenommen. Etwa in den Diskursen zur Zivil- oder Bürgergesellschaft, zum bürgerschaftlichen Engagement oder zu Neuen Sozialen Bewegungen werden affine Fragen nach einem dritten Bereich der Gesellschaft behandelt. Der Gegenstand der vorliegenden Arbeit, die Beschreibung und Analyse des »Nonprofit-Sektors« als distinkten Organisationsbereich neben Erwerbswirtschaft und Staat, verweist dabei gewissermassen auf das

1 SEIBEL (1990): Gibt es einen Dritten Sektor? In: Journal für Sozialforschung

2 Vgl. ANHEIER (1995): Vergleichende Forschung zum Nonprofit-Sektor. In: Schauer u.a., Nonprofit-Organisationen (NPO), S. 29 
organisationale Substrat der Bürgergesellschaft und des bürgerschaftlichen Engagements. Die allermeisten Organisationsformen der Sphäre der Bürgergesellschaft und der überwiegende Teil des organisationsgebundenen bürgerschaftlichen Engagements sind Nonprofit-Organisationen und dem Nonprofit-Sektor zuzurechnen.

Die Forschung zu Nonprofit-Organisationen und dem Nonprofit-Sektor ist heute transdisziplinär - und muss es sein. Wichtige Erkenntnisse, die im Folgenden ausgebreitet werden, kommen aus Soziologie, Politikwissenschaft, Rechtswissenschaft, Ökonomik und Verwaltungswissenschaft. Im Kern handelt es sich jedoch um eine sozialökonomische und soziologischtheoretische Abhandlung. In der Arbeit werden eine ganze Reihe sozialwissenschaftlicher Klassiker zu Wort kommen, zwei theoretische Konzepte werden aber eine besondere Rolle spielen: die soziologische Systemtheorie, insbesondere jene von und um Niklas Luhmann und die Marx'sche politische Ökonomie. Erstere wird herangezogen, um die Systemrationalität und Autonomie des organisationalen Teilsystems Nonprofit-Sektor theoretisch zu begründen; Letztere, weil sie am besten geeignet ist, den Profit-Sektor der Gesellschaft zu verstehen. Die insbesondere von den soziologischen Klassikern rekonstruierte erwerbswirtschaftliche Logik des kapitalistischen Sektors ist dabei die theoretische Referenz für die Beschreibung der Logiken der anderen beiden Sektoren, insbesondere des Nonprofit-Sektors. In Kontrastierung mit der Logik des Profit-Sektors werden damit die Spezifika der anderen beiden Sektoren besonders deutlich.

Die Arbeit wurde im Rahmen eines BMBF-Projekts zu Reorganisationsprozessen im Dritten Sektor an der TU München fertiggestellt (FKZ 01HR9948) und in einer ersten Fassung im Jahr 2002 als Dissertation von der Philosophischen Fakultät der Universität Chemnitz angenommen. Eine Reihe von Personen hat den Entstehungsprozess der Arbeit wohlwollend und kritisch begleitet. Meinem »Doktorvater« G. Günter Voß danke ich vor allem für seine Geduld in der Schlussphase bemühte er sogar mäeutische Verfahren um mitzuhelfen, die zentralen Ergebnisse der Arbeit zu sichern. Ingo Bode verfolgte meine Überlegungen zu Nonprofit-Organisationen über viele Jahre. Ihm verdanke ich nicht nur Literaturhinweise und inhaltliche Anregungen in diversen Diskussionen, sondern auch eine sehr hilfreiche intensive Durchsicht der Rohfassung. Reinhart Lang und Rainer Trinczek erklärten sich freundlicherweise bereit, die Arbeit zu begutachten, und gaben hilfreiche Verbesserungshinweise. Hans Pongratz half immer wieder mit wichtigen Tipps zur Konzeption der Arbeit. Herr Hajo Schneider und Frau Renate Reifferscheid sorgten für eine ordentliche Setzung der Kommata und eine korrekte (neue) Rechtschreibung in schwierigeren Fällen. Herr Witt bot mir freundlicherweise die Publikation in seiner Reihe an. Frau Nicole Schweitzer betreute die Herausgabe der Arbeit von Seiten des Deutschen Universitäts-Verlags und stand auch für ungewöhnliche Fragen und Probleme jederzeit zur Verfügung.

Thomas Wex 


\title{
Inhaltsübersicht
}

\author{
Abschnitt I Grundlegung \\ Nonprofit-Organisationen und der Nonprofit-Sektor
}

Abschnitt II Eine kleine Geschichte der Organisationsgesellschaft

Zur historischen Genese und Entwicklung der Organisationsformen

Abschnitt III Die Morphologie der Organisationsgesellschaft

Organisationssektoren der Gesellschaft

Abschnitt IV Die Idee des Nonprofit-Sektors und die Nonprofit-Forschung

Ein Forschungsüberblick

Abschnitt V Elemente einer Systemtheorie der Organisationsgesellschaft

Abschnitt VI Verwertung - Herrschaft - Assoziation

Die Organisationssektoren und ihre Logiken

Abschnitt VII Zusammenfassung und Schluss

Dritter Sektor als Dritter Weg? 


\section{Inhaltsverzeichnis}

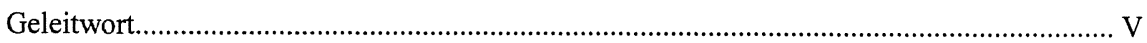

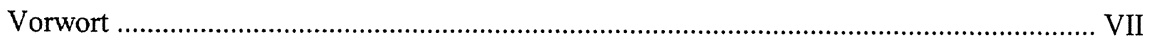

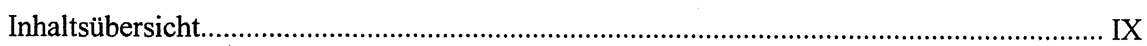

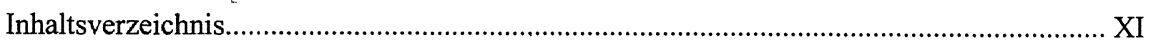

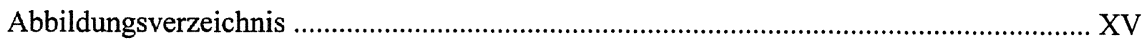

I.

\section{Grundlegung}

Nonprofit-Organisationen und der Nonprofit-Sektor.................................................... 1

1. Die Nonprofit-Organisation ................................................................................... 1

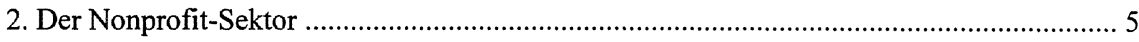

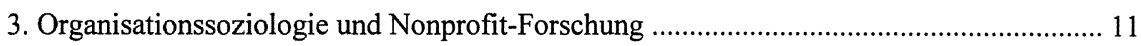

3.1 Organisationssoziologie in Deutschland ............................................................. 10

3.2 Eine Soziologie der Nonprofit-Organisation? ................................................... 13

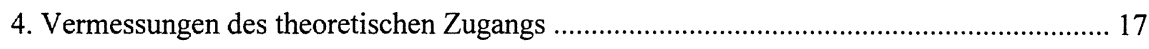

4.1 Nonprofit: Wirtschaftssoziologie vs. Mainstream-Ökonomik ................................. 17

4.2 Eigenlogik: Handlungstheorie vs. Struktur- oder Systemtheorie ............................ 19

4.3 Distinktion: Identitätslogik vs. Differenzlogik ................................................... 22

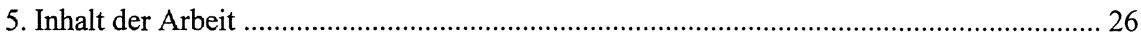

II.

Eine kleine Geschichte der Organisationsgesellschaft -

Zur historischen Genese und Entwicklung der Organisationsformen....................... 31

1. Das Organisationsphänomen moderner Gesellschaften ............................................... 32

1.1 Die moderne Gesellschaft als Organisationsgesellschaft ..................................... 32

1.2 Thematisierungen der Organisationsgesellschaft ................................................ 34

1.3 Freie Vereinigungen als Ursprung aller privater Körperschaften ........................... 42

2. Das historische Vereinswesen als Ursprung des Nonprofit-Sektors ................................. 44

2.1 Frühe Vereinigungen im Einzelnen: Von der Sodalität zur

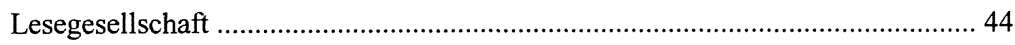

2.2 Ein historischer Abriss der Entwicklung des Vereinswesens .............................. 51

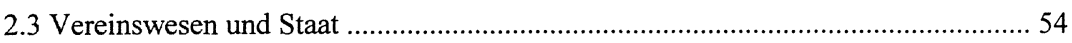

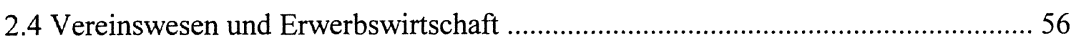


3. Einige (heutige) Rechtsformen des Nonprofit-Sektors in ihrer

historischen Entwicklung .

(Gewerkschaften, unternehmerische Verbände, politische Parteien,

Genossenschaften, Wohlfahrtsverbände)

4. Geschichte erwerbswirtschaftlicher Organisationen .................................................. 71

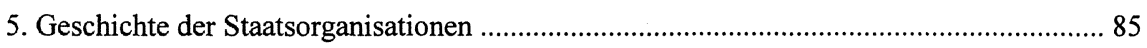

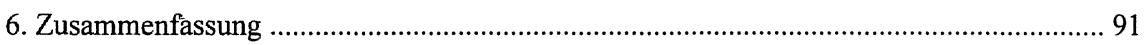

III.

Die Morphologie der Organisationsgesellschaft -

Organisationssektoren der Gesellschaft

1. Die sektorale Perspektive der Organisationsgesellschaft: Ein Modell der

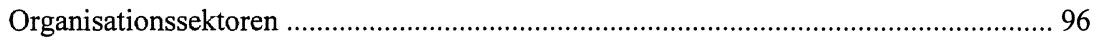

1.1 Ein Modell der Organisationsgesellschaft ............................................................. 96

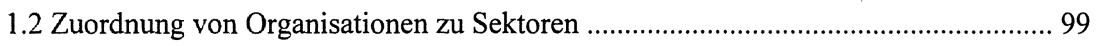

1.3 Verortung von Organisationen innerhalb der Sektoren ........................................... 103

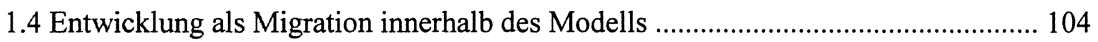

1.5 Übergänge von der informellen Sphäre zu den Organisationssektoren .................... 105

1.6 Interpenetration: Die Überschneidungszonen der Sektoren .................................. 107

2. Einige Organisationstypen des Nonprofit-Sektors im Einzelnen .................................... 108

(Vereine, Exkurs: Gemeinnützigkeit, Wohlfahrtsverbände, Gewerkschaften,

unternehmerische Verbände, Stiftungen, politische Parteien)

3. Zusammenfassung

IV.

Die Idee des Nonprofit-Sektors und die Nonprofit-Forschung:

Ein Forschungsüberblick

1. Entstehung und Entwicklung der Idee eines Nonprofit-Sektors ................................... 124

2. Nonprofit-Forschung im weiteren Sinne nach Disziplinen ........................................... 133

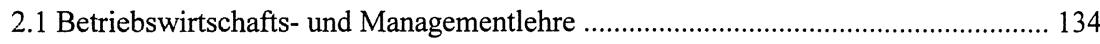

(Genossenschaftswissenschaft, Gemeinwirtschaftslehre,

Bedarfswirtschaft, Verbandsbetriebslehre, öffentliche Wirtschaft,

BWL der Nonprofit-Organisationen, Sozialwirtschaft)

2.2 Volkswirtschaftslehre und Wirtschaftstheorie

(Nonprofit-Ökonomik, Organisationen ohne Erwerbszweck) 
2.3 Soziologie 148

(Freiwillige Vereinigungen, Verbände, Lebensgemeinschaften,

Intermediarität, Öffentlichkeit, Wohlfahrtspluralismus,

Kommunitarismus, Bürgergesellschaft)

2.4 Politikwissenschaft

(Verbände-, Parteienforschung, Johns Hopkins Nonprofit

Forschung, Neue Soziale Bewegungen, Korporatismusforschung,

Nicht-Regierungs-Organisationen - NRO)

2.5 Verwaltungswissenschaft

(Dritte-Sektor-Forschung, verselbstständigte Verwaltungseinheiten,

funktionaler Dilettantismus)

2.6 Sozialpädagogik

(Wohlfahrtsverbände, Sozialmanagement)

2.7 Geschichtswissenschaft 184

(Historische Analyse von Vereinigungen)

2.8 Jura 184

(Gesellschaftsrecht, Rechtsformenlehre)

3. Nonprofit-Forschung im engeren Sinne: Infrastruktur 185

4. Zusammenfassung 190

V.

Elemente einer Systemtheorie der Organisationsgesellschaft........................................... 193

1. Systemtheoretische Analyse der Organisationssektoren ................................................. 194

2. Gesellschaft als soziales System und dessen Struktur ................................................... 199

3. Differenzierung, Verselbstständigung und Imperialismus der Teilsysteme …………....... 206

4. Exkurs: Die Organisationsgesellschaft als kapitalistische Gesellschaft? .......................... 215

5. Autonomie und Selbstorganisation der Teilsysteme ……………………................... 217

6. Interpenetration und Interpenetrationszonen der Teilsysteme ....................................... 228

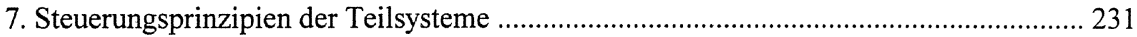

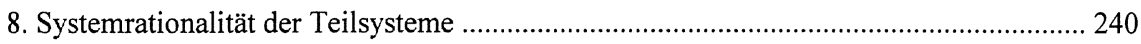

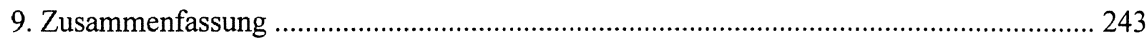


VI.

Verwertung - Herrschaft - Assoziation:

Die Organisationssektoren und ihre Logiken 247

1. Multiple Rationalitäten moderner Gesellschaften -

Logiken gesellschaftlicher Teilbereiche 247

2. Die Logiken der Sektoren im Einzelnen ................................................................... 253

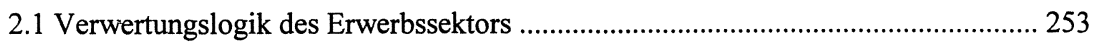

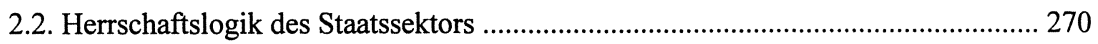

2.3 Assoziationslogik des Nonprofit-Sektors ………............................................. 284

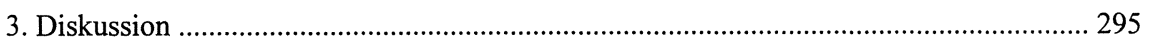

VII.

Zusammenfassung und Schluss 303

Dritter Sektor als Dritter Weg? 


\section{Abbildungsverzeichnis}

1 Typen privater Nonprofit-Organisationen ..................................................... 4, 101

2 Beschäftigung und Freiwilligenarbeit im Nonprofit-Sektor 1990 ……………….......... 6

3 Beschäftigung im Nonprofit-Sektor 1990 und 1995 ................................................... 7

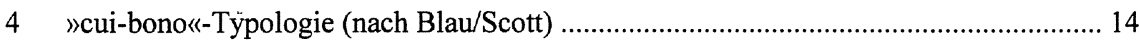

5 A Typology of Compliance Relations (nach Etzioni) ………………………………... 15

6 Wirtschaftssoziologie (Economic Sociology) und . Mainstream Ökonomik - ein Vergleich (nach Smelser/Swedberg) …………………..... 18

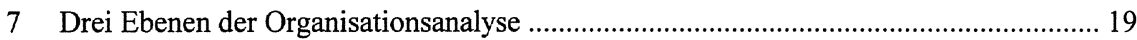

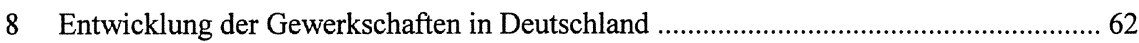

9 Manager-Ideologien („Managerial Ideologies") (nach Perrow) ………………………... 79

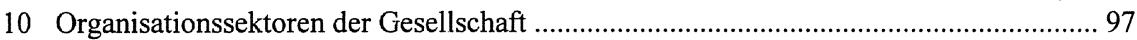

11 Entwicklungsmodelle des Nonprofit-Sektors (nach Salamon/Anheier) .......................... 99

12 Spitzenverbände der freien Wohlfahrtspflege - Struktur und

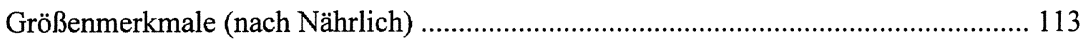

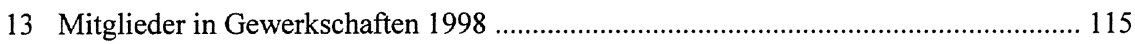

14 Entwicklung der Mitgliederzahlen der Gewerkschaften ............................................ 116

15 Die zehn größten deutschen Stiftungen und ihr Vermögen ......................................... 119

16 Mitgliederentwicklung der politischen Parteien ……………………........................ 121

17 Nonprofit-Forschung nach Disziplinen - eine Übersicht ........................................... 135

18 Ökonomische Ordnung der Organisationsformen (nach Schwarz) …………….......... 142

19 Organisationsformen und ihre ökonomischen Merkmale (nach Schwarz) …………..... 143

20 Distinkte Charakteristika von Nonprofit-Organisationen (nach Mason) ……................ 144

21 Ökonomische Nonprofit-Sektor-Theorien im Überblick (nach Anheier)......................... 150

22 Modelle sozialer Ordnung (bei Streeck/Schmitter) …………………........................ 155

23 Organisationen im „Welfare Triangle“ (nach Evers) ................................................. 158

24 Merkmale der Sektoren der Wohlfahrtsproduktion (nach Evers/Olk) ......................... 163

25 Grundriß der bürgerlichen Öffentlichkeit im 18. Jahrhundert.

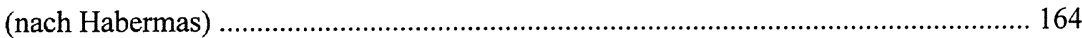

26 Strukturbesonderheiten von Vereinen und Unternehmen/Firmen

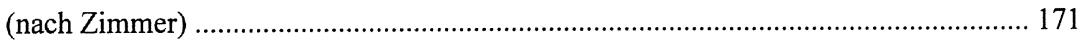

27 Typologie von Vereinigungen (nach Alemann) ………......................................... 172

28 Gesellschaftliche Verortung des Nonprofit-Sektors (nach Reichard) ……………....... 175

29 Das Dritte-Sektor-Konzept (von Schuppert) .......................................................... 178

30 Abstufungen privater und staatlicher Aufgabenwahrnehmung

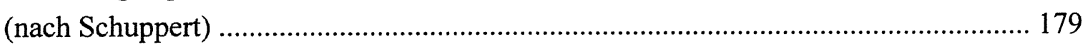

31 Klassifikationen von Steuerungsformen - ein Überblick ............................................. 234 
32 Soziale Logiken im Drei-Ebenen-Modell 251

33 Politische Themenkonjunkturen der Staatsdiskussion (nach Jann) 277

34 Das Gegenstromprinzip der Führung in Nonprofit-Organisationen (nach Schwarz)

35 Dominante sozioökonomische Prinzipien der Organisationen der Erwerbswirtschaft und des Nonprofit-Sektors

36 Ökonomische Strukturmerkmale der Organisationen der Erwerbswirtschaft, des Staates und des Nonprofit-Sektors

37 Vergleich der Commons, des Marktes und des Staates (nach Lohmann) 294

38 Die assoziative, ökonomische und herrschaftliche Dimension der Gesellschaft und ihrer Sektoren 